\title{
The association between the angiotensin-converting enzyme- 2 gene and blood pressure in a cohort study of adolescents
}

Lucile Malard ${ }^{1,2}$, Lisa Kakinami ${ }^{1}$, Jennifer O'Loughlin ${ }^{3}$, Marie-Hélène Roy-Gagnon ${ }^{4,5}$, Aurélie Labbe ${ }^{1}$, Louise Pilote ${ }^{6}$, Pavel Hamet ${ }^{7}$, Johanne Tremblay ${ }^{7}$ and Gilles Paradis $^{1^{*}}$

\begin{abstract}
Background: The Angiotensin-Converting Enzyme-2 (ACE2) gene, located on chromosome X, is believed to be implicated in blood pressure regulation. However the few studies that have examined this association have yielded mixed results. The objective of this study was to assess the association between tag single nucleotide polymorphisms (SNPs) in the angiotensin-converting enzyme-2 gene with blood pressure and blood pressure change in adolescents.

Methods: Participants in the Nicotine Dependence in Teens (NDIT) cohort study with blood or saliva samples and at least 3 blood pressure measurements over 5 years were included in the analytic sample $(n=555)$. Linear growth curve models stratified on sex and ethnicity were used to assess the association between four tag SNPs in the ACE2 gene and systolic (SBP) and diastolic blood pressure (DBP), and blood pressure change.

Results: In males of European descent, rs2074192 and rs233575 were significantly associated with SBP and DBP, and rs2158083 was associated with SBP. In French Canadian males, rs233575 and rs2158083 were significantly associated with DBP. Among females of European descent, rs2074192, rs233575, and rs2158083 were significantly associated with change in SBP over 5 years.

Conclusions: This is the first study to assess the association between the ACE2 gene with blood pressure and blood pressure change in a cohort of adolescents. Results indicate that several ACE2 gene SNPs are associated with blood pressure or blood pressure change in persons of European descent. However the therapeutic potential of these SNPs should be explored.
\end{abstract}

Keywords: Blood pressure, Angiotensin-Converting Enzyme-2, Adolescent, Cohort study

\section{Background}

Hypertension (HTN) is a major risk factor for cardiovascular disease. Half of all strokes and ischemic heart disease, and $25 \%$ of other cardiovascular diseases are due to elevated blood pressure [1]. In 2000, the prevalence of HTN as defined by systolic blood pressure (SBP) $\geq 140 \mathrm{mmHg}$ or diastolic blood pressure (DBP) $\geq 90 \mathrm{mmHg}$ was $26 \%$ worldwide [2]. It is estimated that the prevalence will reach $29 \%$ by 2025 [2]. Genetic influences on blood pressure have been clearly established, with heritability estimates between

\footnotetext{
*Correspondence: gilles.paradis@mcgill.ca

'Department of Epidemiology, Biostatistics and Occupational Health, McGill University, Montréal, QC, Canada

Full list of author information is available at the end of the article
}

0.20 and 0.60 [3-6]. It is thus important to better understand which genes are implicated in the regulation of blood pressure and how these genes influence blood pressure levels. Increased understanding of genetic risk could help identify new therapeutic targets for HTN, as well as distinguish population subgroups that may benefit from closer monitoring and more intensive prevention efforts.

The Angiotensin-Converting Enzyme-2 (ACE2) is a negative regulator of the renin-angiotensin aldosterone system [7-9] and plays an important role in blood pressure homeostasis [9]. ACE2, a homolog to the Angiotensin-Converting Enzyme (ACE), contains a single catalytic site that is $42 \%$ similar to the ACE catalytic sites, and ACE2 and ACE are believed to have antagonistic effects on blood pressure

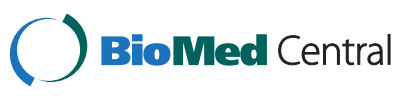


$[7,8,10,11]$. While animal models demonstrate an increase in Ang II peptide levels (a vasoconstrictor substance) with ACE2 inhibition [9,12], studies of the association between blood pressure and ACE2 single nucleotide polymorphisms (SNPs) have yielded mixed results in humans. Studies in Australian, German, and Han Chinese populations did not find any association between ACE2 SNPs and blood pressure $[11,13,14]$, while studies in Chinese populations that were not Han Chinese found statistically significant associations between two ACE2 SNPs and blood pressure $[15,16]$.

Past studies on ACE2 SNPs were conducted in adults and were primarily case-control or cross-sectional. The results are subject to confounding by preexisting chronic diseases or treatments for high blood pressure. Because young people generally do not have chronic disease or take blood pressure medications, assessing the association between blood pressure and ACE2 using data from adolescents may improve our understanding of the implication of this gene in blood pressure regulation. Thus, the aim of this study was to assess the association between blood pressure and ACE2 gene SNPs in an ongoing cohort study of healthy adolescents.

\section{Methods}

\section{Study population}

The Nicotine Dependence in Teens (NDIT) Study is an ongoing prospective cohort investigation designed to assess the development of nicotine dependence symptoms after cigarette smoking onset in adolescents [17]. Participants were recruited in a convenience sample of ten French and English schools in Montreal. Schools were located in urban, suburban and rural settings, and participants were of varying socio-economic status. A total of 1,293 students in grade 7 (12-13 years of age) participated in the baseline assessment in 1999-2000.

Participants completed 45-minute self-reported questionnaires every 3-4 months during the 10-month school year, for a total of 20 waves of data collection during high school. Data from the baseline and the first five years of follow-up were used in this study. Blood pressure and anthropometric measures were obtained in survey cycle 1, 12 and 19 during secondary school (when participants were 12.7, 15.1, and 17.0 years of age on average, respectively). The blood or saliva samples for DNA extraction were provided by 852 of the 1293 participants (65.9\%) in 2002 and 2007. All participants and a parent or guardian provided written informed consent at baseline; a separate consent was provided by parents for the blood draw in grade 9 (when participants were 14-15 years of age), and informed consent was obtained from participants (who had attained legal age) for saliva samples in 2007. The study was approved by Institutional Review Boards at the CRCHUM, the Direction de santé publique de Montreal and McGill University.

\section{Measurements}

After voiding and a 5-minute rest, blood pressure was measured on the right arm of seated subjects according to a standardized protocol by a trained and certified technician. Measurements were made with an automated oscillometric device (Dinamap XL, model CR9340; Critikon Company LLC, Tampa, Florida) calibrated against a mercury sphygmomanometer before each data collection period. SBP and DBP were measured 3 times at 1-minute intervals. If the SBP and DBP measurements differed by more than 10 and $5 \mathrm{mmHg}$ respectively, fourth and fifth measurements were obtained. The mean of the two closest measurements (after exclusion of the first measurement) was used for analysis.

\section{Covariates}

Duplicate measurements of height and weight were obtained with participants wearing light clothing without shoes, using a stadiometer (model 214 Road Rod; Seca Corp., Hanover, MD, USA) for height and a scale (floor model 761; Seca Corp., Hanover, MD, USA) for weight. A third measurement was obtained if the difference between the two measurements was greater than $0.5 \mathrm{~cm}$ for height and $0.2 \mathrm{~kg}$ for weight. The average of the two closest measurements was used in the analysis. To assess inter-rater reliability of height and weight, measures of every 1 in 10 participants were repeated. Inter-rater reliabilities were 0.99 for height and weight $[18,19]$. Sexand age-specific body mass index (BMI) percentiles were assessed and youth were categorized as overweight or obese if the BMI was $\geq 85$ th percentile according to the Centers for Disease Control and Prevention guidelines [20]. The date of birth and sex of participants were selfreported in the questionnaire.

\section{SNP selection and genotyping}

We used the greedy pairwise tagging approach [21] as implemented in the software Haploview [22] in order to tag polymorphisms present in the HapMap CEU data. We selected SNPs with a minor allele frequency $>5 \%$. We used a linkage disequilibrium (LD) cutoff of $\mathrm{r} 2>0.8$, ensuring that each unmeasured SNP is in strong LD with a tag SNP. We tagged polymorphisms from $10 \mathrm{~kb}$ before to $10 \mathrm{~kb}$ after the location of ACE2 using genomic positions according to NCBI build 36. The four selected tag SNPs (rs2074192, rs233575, rs2158083 and rs1978124) were genotyped at the McGill University and Genome Quebec Innovation Center using iPLEX Gold technology (Sequenom, San Diego, Calif, USA).

\section{Data analysis}

Participants with only one or two blood pressure measurements during the five years of high school were excluded from analyses because of the potential for over fitting. Because females begin puberty before males, and 
the evolution of blood pressure differs between males and females in adolescence [23,24], the analyses were stratified by sex. Differences in demographic and baseline characteristics between males and females were tested with chi-squares and t-tests, and differences in SBP or DBP change over time were tested with general linear models and paired t-tests as appropriate. Because the ACE2 gene is located on the X chromosome, males have only one allele of the ACE2 gene while females have two. However, which of the alleles is expressed is unknown in heterozygous females, and therefore we restricted our analyses to males and homozygous females for each specific SNP, as has been done in the literature [25]. We tested Hardy-Weinberg Equilibrium in females. Differences in allele frequencies between males and females and LD were assessed with $X^{2}$ tests.

Due to evidence of heterogeneity between ethnicities, analyses were also stratified by ethnicity (French Canadian, European, Other). Analyses assessing the association between baseline blood pressure and the major and minor SNP genotypes were conducted with linear regression analyses. Major and minor SNP genotypes were identified in accordance with the dbSNP database [26]. A linear growth curve model with an unstructured error covariance structure was used to assess the association between SBP or DBP, and change of SBP or DBP with the four ACE2 SNPs. SBP and DBP were modeled as continuous variables in separate models; time was represented by age centered at 12 years, ethnicity was defined as French Canadian, European descent, or Other (biological parents from Asian, Spanish, African, etc., countries), and the major allele genotypes served as the reference category (GG for rs2074192 and rs1978124, and TT for rs233575 and rs2158083 for females; G for rs2074192 and rs1978124, and T for rs233575 and rs2158083 for males). All models adjusted for height and whether or not the participant was overweight or obese. Statistical analyses were carried out using SAS 9.2 software (SAS Institute Inc., Cary, NC, USA).

\section{Results}

Participants with fewer than three blood pressure measurements $(n=570)$, or those who did not provide a blood or saliva sample $(n=168)$ were excluded. Therefore a total of 555 participants of the original 1,293 were retained for analyses. At baseline, adolescents who were excluded were significantly younger than those retained in analyses (12.6 years old compared to $12.9, p<0.05$ ) but did not differ in sex, ethnicity, whether or not they were overweight or obese, SBP or DBP $(p>0.05$, data not shown).

Over half (52\%) of the participants in the complete sample $(n=555)$ were male (Table 1$)$. Females were not significantly different from males in age, ethnicity, BMI, or baseline SBP or DBP. Mean SBP at baseline was 105
$\mathrm{mmHg}$ and $104 \mathrm{mmHg}$ in males and females, respectively and mean baseline DBP was $56 \mathrm{mmHg}$ and $56 \mathrm{mmHg}$ in males and females respectively. SBP was moderately correlated with DBP in wave 1,12 , and 19 (Pearson $r=0.55$, 0.59 , and 0.59 , respectively). These results were unchanged after excluding heterozygote females (data not shown). SBP was statistically significantly lower in females than males at both follow-ups $(p<0.0001$ in wave 12 and wave 19), but DBP was not statistically significantly different $(p>0.05$ in wave 12 and wave 19). SBP increased $10.1 \mathrm{mmHg}$ on average over the 5 -year follow-up $(p<0.0001)$ in males, and increased $2.0 \mathrm{mmHg}$ on average in females $(p=0.0003)$, a statistically significant difference $(p<0.0001)$. DBP increased $3.4 \mathrm{mmHg}$ on average over the 5 -year follow-up in males $(p<0.0001)$, and $2.2 \mathrm{mmHg}$ on average in females $(p<0.0001)$, but the difference was not statistically significant by sex $(p>0.05)$. These results were unchanged after excluding heterozygote females (data not shown).

As mentioned in our methods, we excluded heterozygous females from analyses for each specific SNP resulting in analytic samples that ranged from 407-415 participants. Allele frequencies stratified by ethnicity were compared between males and females and were not statistically significantly different (Table 2), except for rs2074192 which had an A allele frequency of $25 \%$ in French Canadian males compared to $44 \%$ in French Canadian females $(p=0.01)$, and an A allele frequency of $27 \%$ in males of other ethnicity compared to $41 \%$ in females of other ethnicity $(p=0.01)$. SNPs rs233575, rs2158083, and rs1978124 were in Hardy-Weinberg Equilibrium ( $p>0.05$, data not shown). All SNPs were in linkage disequilibrium with each other (Additional file 1: Table S1).

\section{SNPs and baseline blood pressure}

In multivariable linear regression analyses, SNP rs1978124 was not associated with SBP or DBP at baseline in either males or females $(p>0.05$ for all, Additional file 2: Table S2). Among males of European descent, carrying the G allele of SNP rs2074192 was associated with higher DBP at baseline ( $56 \mathrm{mmHg}$ vs $53 \mathrm{mmHg}, p<0.05$ ), and the T allele of SNP rs233575 was associated with lower SBP (104 $\mathrm{mmHg}$ vs $108 \mathrm{mmHg}, p<0.05)$ and DBP $(54 \mathrm{mmHg}$ vs 56 $\mathrm{mmHg}, p<0.05)$. French Canadian females carrying the $\mathrm{T}$ allele for rs 233575 or rs2158083 had baseline DBP that was $4 \mathrm{mmHg}$ higher $(59 \mathrm{mmHg}$ vs $55 \mathrm{mmHg}$ ) than French Canadian females carrying the $\mathrm{C}$ allele $(p<0.05)$.

\section{SNPs and blood pressure, or SNPS and blood pressure change (males)}

Sex- and ethnicity-stratified analyses suggested that after adjusting for height and weight status, SBP and DBP in males of European descent carrying the A allele of rs2074192 were $3.3 \mathrm{mmHg}$ lower $(p=0.02)$ and 2.3 
Table 1 Selected characteristics $(n=555)$ of the NDIT participants, 1999-2005

\begin{tabular}{|c|c|c|c|}
\hline Characteristic $^{1}$ & $\begin{array}{c}\text { Male } \\
(n=265)\end{array}$ & $\begin{array}{c}\text { Female } \\
(n=290)\end{array}$ & $p^{2}$ \\
\hline$\overline{\text { Age, years, mean }(S D)^{3}}$ & $12.7(0.42)$ & $12.6(0.39)$ & 0.06 \\
\hline \multicolumn{4}{|l|}{ Ethnicity, \% } \\
\hline French Canadian & 29 & 32 & 0.71 \\
\hline European descent & 47 & 43 & \\
\hline Other & 24 & 25 & \\
\hline Overweight or obese, $\%$ & 27 & 21 & 0.11 \\
\hline \multicolumn{4}{|l|}{ SBP, mmHg, mean (SD) } \\
\hline Baseline ( 13 years of age) & $105.3(10.2)$ & $104.5(9.7)$ & 0.36 \\
\hline Wave 12 ( 15 years of age) & $109.1(11.2)$ & $104.1(10.0)$ & $<0.0001$ \\
\hline Wave 19 ( 17 years of age) & $115.4(11.5)$ & $106.6(10.4)$ & $<0.0001$ \\
\hline \multicolumn{4}{|l|}{$\mathrm{DBP}, \mathrm{mmHg}$, mean (SD) } \\
\hline Baseline ( 13 years of age) & $55.6(6.0)$ & $56.5(6.2)$ & 0.08 \\
\hline Wave 12 ( 15 years of age) & $56.7(5.7)$ & $57.4(6.5)$ & 0.19 \\
\hline Wave 19 ( 17 years of age) & $58.9(6.3)$ & $58.6(6.3)$ & 0.58 \\
\hline
\end{tabular}

${ }^{1}$ Baseline measurements unless otherwise noted; ${ }^{2}$ difference between males and females; ${ }^{3}$ SD: Standard deviation.

mmHg lower $(p=0.003)$, respectively than in males of European descent carrying the $\mathrm{G}$ allele (Table 3). In contrast, the SBP and DBP of males of European descent carrying the rs $233575 \mathrm{C}$ allele were $3.6 \mathrm{mmHg}(p=0.01)$ and $2.4 \mathrm{mmHg}$ higher $(p=0.003)$, respectively, than in males of European descent carrying the $\mathrm{T}$ allele. In addition, males carrying the $\mathrm{C}$ allele of rs 2158083 had SBP that was $3.4 \mathrm{mmHg}$ higher $(p=0.02)$ than males carrying the $\mathrm{T}$ allele. French-Canadian males with the $\mathrm{C}$ allele of rs233575 and rs2158083 had $2.9 \mathrm{mmHg}$ $(p=0.001)$ and $2.7 \mathrm{mmHg}(p=0.01)$ lower DBP than males with the $\mathrm{T}$ allele of these respective SNPs. There were no statistically significant associations between any of the four SNPs and change in SBP or DBP during follow-up among boys (data not shown).

\section{SNPs and blood pressure, or SNPS and blood pressure change (females)}

In general none of the SNPs were associated with either SBP or DBP in females after adjustment for covariates (data not shown). However, there were statistically significant associations between several SNPs and change in SBP among females (Table 4). Females of European descent carrying the A allele of rs2074192 had a change in SBP during follow-up that was $1.5 \mathrm{mmHg}$ lower than females of European descent carrying the $\mathrm{G}$ allele $(p=0.002)$. Females of European descent carrying the $\mathrm{C}$ allele of rs 233575 and rs2158083 had a change in their SBP that was $1.9 \mathrm{mmHg}$ $(p=0.002)$ and $1.20 \mathrm{mmHg}(p=0.04)$ higher, respectively, compared to females of European descent carrying the $\mathrm{T}$ allele.

\section{Discussion}

As a part of the renin-angiotensin aldosterone system, an important regulator of blood pressure homeostasis, ACE2 is believed to have a role in blood pressure regulation [9]. Indeed, ACE2 catalyzes the conversions of Angiotensin I and II into Angiotensin 1-7 which is known to have a vasodilator function $[7,8]$. Moreover, strong biological evidence from animal models suggests that the ACE2 gene is a regulator of blood pressure [7-12]. However, the human genetic literature is mixed with some studies reporting a relationship between ACE2 SNPs and blood pressure $[15,16]$ while others do not $[11,13,14]$.

To the best of our knowledge, our study is the first to investigate the association between tag SNPs in the ACE2 gene with blood pressure and blood pressure change in adolescents using multiple measures of blood pressure over five years of follow-up. Interestingly, we observed two associations (rs2074192 and rs233575) in males and females that were entirely driven by participants of European ancestry. Our results indicate that the ACE2 gene and more specifically, the major allele of rs2074192 and the minor allele of rs233575 significantly increase SBP and DBP among males of European ancestry, and significantly increase SBP change among females of European ancestry.

All previous studies on the ACE2 gene were carried out in adults using cross-sectional or case-control designs with single-point estimates, or follow-up time less than a month. In contrast, our study utilized nearly five years of follow-up among adolescents. The use of

Table 2 Frequency of the minor alleles stratified by sex and ethnicity (NDIT Study, 1999-2005)

\begin{tabular}{|c|c|c|c|c|c|c|c|c|c|}
\hline \multirow[b]{2}{*}{ SNP (Minor/Major) ${ }^{1,2}$} & \multicolumn{3}{|c|}{ French Canadian } & \multicolumn{3}{|c|}{ European } & \multicolumn{3}{|c|}{ Other } \\
\hline & Male & Female & $p^{3}$ & Male & Female & $p^{3}$ & Male & Female & $p^{3}$ \\
\hline rs2074192 (A/G) & $24.7 \%$ & $43.9 \%$ & $<0.01$ & $47.1 \%$ & $46.8 \%$ & 0.93 & $26.6 \%$ & $40.7 \%$ & 0.01 \\
\hline rs233575 (C/T) & $41.9 \%$ & $36.8 \%$ & 0.35 & $37.5 \%$ & $39.9 \%$ & 0.58 & $33.3 \%$ & $23.2 \%$ & 0.07 \\
\hline rs2158083 (C/T) & $37.7 \%$ & $37.5 \%$ & 0.98 & $40.3 \%$ & $40.4 \%$ & 0.99 & $33.3 \%$ & $23.9 \%$ & 0.09 \\
\hline rs1978124 (A/G) & $45.4 \%$ & $49.4 \%$ & 0.46 & $55.4 \%$ & $52.0 \%$ & 0.46 & $31.2 \%$ & $31.0 \%$ & 0.96 \\
\hline
\end{tabular}

${ }^{1}$ Depending on the SNP, $n$ ranged from 142 to 150 female, and 257 to 264 male participants; ${ }^{2}$ Categorizations of major and minor genotypes in accordance with dbSNP database; ${ }^{3}$ Comparison of frequencies between males and females. 
Table 3 Association between minor ACE2 alleles and blood pressure differences among males (NDIT Study, 1999-2005)

\begin{tabular}{|c|c|c|c|c|c|c|}
\hline \multirow[b]{3}{*}{$\mathrm{SNP}^{2}$} & \multicolumn{3}{|c|}{$\mathrm{SBP}, \mathrm{mmHg}$} & \multicolumn{3}{|c|}{$\mathrm{DBP}, \mathrm{mmHg}$} \\
\hline & \multicolumn{3}{|c|}{ Beta (Confidence interval) ${ }^{1}$} & \multicolumn{3}{|c|}{ Beta (Confidence interval) $^{1}$} \\
\hline & French Canadian & European & Other & French Canadian & European & Other \\
\hline rs2074192 & $-2.65(-7.3,2.0)$ & $-3.32(-6.1,-0.6)^{3}$ & $1.57(-3.5,6.6)$ & $0.41(-2.0,2.8)$ & $-2.32(-3.8,-0.8)^{4}$ & $-1.18(-4.1,1.7)$ \\
\hline rs233575 & $-1.05(-5.3,3.2)$ & $3.59(0.8,6.4)^{5}$ & $-1.05(-5.9,3.8)$ & $-2.88(-5.0 .-0.8)^{6}$ & $2.42(0.8,4.0)^{7}$ & $-1.24(-4.0,1.6)$ \\
\hline rs2158083 & $-1.85(-6.0,2.3)$ & $3.40(0.6-6.2)^{8}$ & $-0.32(-5.2,4.6)$ & $-2.66(-4.8 .-0.6)^{9}$ & $1.33(-0.3-2.9)$ & $-0.20(-3.0,2.6)$ \\
\hline rs1978124 & $-2.01(-6.0,2.0)$ & $2.00(-0.8,4.8)$ & $1.79(-3.0,6.6)$ & $-1.29(-3.3,0.8)$ & $0.73(-0.9,2.4)$ & $0.90(-1.9,3.7)$ \\
\hline
\end{tabular}

${ }^{1}$ Adjusted for height, and whether or not the participant was overweight or obese; ${ }^{2}$ Reference groups were the major genotypes in accordance with dbSNP database: $\mathrm{G}$ for rs2074192 and rs $1978124 ; \mathrm{T}$ for rs 233575 and rs $2158083 ;{ }^{3} p$-value $=0.02 ;{ }^{4} p$-value $=0.003 ;{ }^{5} p$-value $=0.01 ;{ }^{6} p$-value $=0.001 ;{ }^{7} p$-value $=0.003$; ${ }^{8} p$-value $=0.02 ;{ }^{9} p$-value $=0.01$.

multiple blood pressure measurements in longitudinal data are less affected by random sources of variation than measurements taken at one time [27] and thus can provide stronger evidence for an association. Consistent with an intervention study among Han Chinese at highrisk for hypertension [28], rs2074192 was statistically significantly associated with DBP and SBP among the males in our study. Consistent with previous studies in German and Han Chinese populations, our results did not detect any associations between blood pressure and rs1978124 [11,14].

Our study is not without limitations. Candidate gene studies and Genome Wide Association Studies have both been criticized for their low replication of results, and candidate gene studies are additionally criticized for the subjectivity of their gene selection [29]. However, we selected the ACE2 gene based on animal models as well as the role of the gene in blood pressure homeostasis, and our results are consistent with previous studies conducted among adults. While candidate gene studies cannot provide an expansive analysis of all possible causative genes, they can provide insight into our understanding of biological pathways [30]. However, our study was limited to tag-SNPs of the ACE2 gene and future candidate gene studies would benefit from replication in larger studies. Due to restricting our analyses to homozygous females and stratifying by sex and ethnicity, our sample size varied between analyses and may be underpowered. Nevertheless, although we did not adjust for multiple testing, we found significant associations between tag SNPs and systolic blood pressure change among females that were consistent with the systolic blood pressure differences among males.

We acknowledge that restricting the analysis to homozygous females discards some information and can lead to a loss of power. Clayton $(2008,2009)$ has suggested tests of associations for SNPs located on the X chromosome that take into account $\mathrm{X}$ inactivation by giving a weight of $1 / 2$ to the heterozygous genotype in females, essentially using an additive coding for the genotype (i.e., genotypes are coded as 0,1 , or 2 for the number of minor alleles) [31,32]. We analyzed our data using this model in females and obtained very similar results as those obtained by comparing the two homozygous genotypes (Additional file 3: Table S3 and Additional file 4: Table S4). We also used other models of $\mathrm{X}$ inactivation, corresponding to dominant (Additional file 5: Table S5 and Additional file 6: Table $\mathrm{S6}$ ) and recessive coding of the genotypes (Additional file 7: Table S7 and Additional file 8: Table S8) [33], and obtained similar results. Given the uncertainty in the model of $\mathrm{X}$ inactivation, we feel that it is more conservative to present our analyses excluding heterozygous females.

While $92 \%$ of our participants were Canadian-born, 28\% of their mothers and $37 \%$ of their fathers were foreignborn, and ethnicity has been shown to modify the association between SNPs and blood pressure [34]. Our tag SNPs were selected based on the HapMap data of northern and western European ancestry, which may not have provided sufficient coverage in other ethnicities. However, the "Other" ethnicity group was heterogeneous, and there were

Table 4 Association between minor ACE2 alleles and blood pressure change among females (NDIT Study, 1999-2005)

\begin{tabular}{|c|c|c|c|c|c|c|}
\hline \multirow[b]{3}{*}{ SNP $^{2}$} & \multicolumn{3}{|c|}{ SBP, mmHg change } & \multicolumn{3}{|c|}{ DBP, mmHg change } \\
\hline & \multicolumn{3}{|c|}{ Beta (Confidence interval) ${ }^{1}$} & \multicolumn{3}{|c|}{ Beta (Confidence interval) ${ }^{1}$} \\
\hline & French Canadian & European & Other & French Canadian & European & Other \\
\hline rs2074192 & $-0.59(-1.8,0.6)$ & $-1.54(-0.6,-2.5)^{3}$ & $-1.48(-3.2,0.2)$ & $-0.44(-1.5,0.6)$ & $-0.20(-1.0,0.6)$ & $-0.32(-1.7,1.0)$ \\
\hline rs 233575 & $0.30(-1.3,1.9)$ & $1.95(0.8,3.1)^{4}$ & $1.41(-0.6,3.4)$ & $0.87(-0.3,2.0)$ & $0.67(-0.1,1.4)$ & $-0.35(-2.0,1.3)$ \\
\hline rs2158083 & $-0.05(-1.4,1.3)$ & $1.20(0.1,2.3)^{5}$ & $2.83(-0.2,5.9)$ & $0.39(-0.7,1.5)$ & $0.53(-0.2,1.2)$ & $0.06(-2.3,2.4)$ \\
\hline rs1978124 & $0.34(-1.0,1.7)$ & $0.78(-0.3,1.9)$ & $0.04(-1.4,1.5)$ & $-0.25(-1.3,0.8)$ & $0.62(-0.01,1.2)$ & $-0.45(-1.6 .0 .7)$ \\
\hline
\end{tabular}

${ }^{1}$ Adjusted for height, and whether or not the participant was overweight or obese; ${ }^{2}$ Reference groups were the major genotypes in accordance with dbSNP database: $\mathrm{G}$ for rs 2074192 and rs $1978124 ; \mathrm{T}$ for rs 233575 and rs $2158083 ;{ }^{3} p$-value $=0.002 ;{ }^{4} p$-value $=0.002 ;{ }^{5} p$-value $=0.04$. 
too few participants of any one ethnicity to warrant selecting tag SNPs. While insufficient coverage could contribute to the absence of association detected in the "Other" ethnicity group, significant results in this group would be extremely hard to interpret given the possibility of population stratification bias. Although most SNPs were in HardyWeinberg Equilibrium, there was evidence of a significant difference in the allele frequencies of rs2074192 between males and females of "French-Canadian" and "Other" descent. Lastly, puberty in females ends at approximately 15 years of age, and it is possible that blood pressure trajectories change at this time. However we were not able to assess this given that we only had three blood pressure measurements over time. Additional studies allowing for two different slopes in females (before and after age 15 years) may provide additional insight.

\section{Conclusion}

Despite these limitations, our results suggest that several ACE2 gene SNPs are associated with either blood pressure levels or blood pressure change among males and females of European ancestry. Whether these SNPs could be new therapeutic targets, or potential primordial targets in persons of European descent should be further explored.

\section{Additional files}

Additional file 1: Table S1. Linkage disequilibrium between SNPs in homozygous females (NDIT Study, 1999-2005).

Additional file 2: Table S2. Mean blood pressure $(\mathrm{mmHg})$ at baseline according to genotype among homozygous participants (NDIT Study, 1999-2005)

Additional file 3: Table S3. Association between minor ACE2 alleles and blood pressure differences among females (NDIT Study, 1999-2005) using the additive model.

Additional file 4: Table S4. Association between minor ACE2 alleles and blood pressure change among females (NDIT Study, 1999-2005) using the additive model.

Additional file 5: Table S5. Association between minor ACE2 alleles and blood pressure differences among females (NDIT Study, 1999-2005) using the dominant model.

Additional file 6: Table S6. Association between minor ACE2 alleles and blood pressure change among females (NDIT Study, 1999-2005) using the dominant model.

Additional file 7: Table S7. Association between minor ACE2 alleles and blood pressure differences among females (NDIT Study, 1999-2005) using the recessive model

Additional file 8: Table S8. Association between minor ACE2 alleles and blood pressure change among females (NDIT Study, 1999-2005) using the recessive model.

\section{Abbreviations}

ACE2: Angiotensin-converting enzyme-2; BMI: Body mass index: DBP: Diastolic blood pressure; HTN: Hypertension; NDIT: Nicotine dependence in teens study; SBP: Systolic blood pressure; SNPS: Single nucleotide polymorphisms.

\section{Competing interests}

The authors have no competing interests to declare.

\section{Author's contributions}

LM performed the statistical analysis, interpreted the data, and drafted the manuscript. LK contributed to the interpretation of the data and participated in the drafting of the manuscript. JOL conceived of and designed the study, obtained the funding, participated in its coordination, contributed to the interpretation of the data, and participated in the drafting of the manuscript. $L P, P H, J T$, conceived of and designed the study, obtained the funding, contributed to the interpretation of the data, and participated in the drafting of the manuscript. GP participated in the conception of and design of the study, contributed to the interpretation of the data, and participated in the drafting of the manuscript. M-H R-G and AL contributed to the statistical analysis, interpretation of the data and participated in the drafting of the manuscript. All authors read and approved the final manuscript.

\section{Acknowledgements}

This research was funded in part by GENESIS, an Interdisciplinary Capacity Enhancement team of the Canadian Institute of Health Research (CIHR) and the Heart and Stroke Foundation of Canada, and by the Canadian Cancer Society. GP holds a CIHR Applied Public Health Research Chair, and JOL holds a Canada Research Chair in the Early Determinants of Adult Chronic Disease. LP holds a James McGill chair at McGill University. Portions of this manuscript have been presented at the American Heart Association Epidemiology and Prevention, Nutrition, Physical Activity and Metabolism Scientific Sessions in San Diego, CA March 13-16, 2012.

\section{Author details}

${ }^{1}$ Department of Epidemiology, Biostatistics and Occupational Health, McGill University, Montréal, QC, Canada. ${ }^{2}$ Institut de Santé Publique d'Epidémiologie et de Développement (ISPED), Université Bordeaux Segalen, Bordeaux, France. ${ }^{3} \mathrm{CRCHUM}$, Département de médecine sociale et préventive, Université de Montréal, Montréal, QC, Canada. ${ }^{4}$ Sainte-Justine University Hospital Research Center, Université de Montréal, Montréal, QC, Canada. ${ }^{5}$ Department of Epidemiology and Community Medicine, University of Ottawa, Ottawa, ON, Canada. ${ }^{6}$ Department of Medicine, McGill University and McGill University Health Center, Montréal, QC, Canada. ${ }^{7}$ Department of Medicine, Centre de recherche du CHUM, Université de Montréal, Montréal, QC, Canada.

Received: 17 January 2013 Accepted: 30 October 2013

Published: 5 November 2013

\section{References}

1. Lawes CMM, Hoorn SV, Rodgers A: Global burden of blood-pressurerelated disease, 2001. Lancet 2008, 371(9623):1513-1518.

2. Kearney PM, Whelton M, Reynolds K, Muntner P, Whelton PK, He J: Global burden of hypertension: analysis of worldwide data. Lancet 2005, 365(9455):217-223

3. Van Rijn MJE, Schut AF, Aulchenko Y, Deinum J, Sayed-Tabatabaei FA, Yazdanpanah M, Isaacs A, Axenovich TI, Zorkoltseva IV, Zillikens MC, et al: Heritability of blood pressure traits and the genetic contribution to blood pressure variance explained by four blood-pressure-related genes. J Hypertens 2007, 25(3):565-570.

4. Pilia G, Chen W-M, Scuteri A, Orrú M, Albai G, Dei M, Lai S, Usala G, Lai M, Loi $P$, et al: Heritability of cardiovascular and personality traits in 6,148 Sardinians. PLoS Genet 2006, 2(8):e132.

5. Snieder H, Harshfield GA, Treiber FA: Heritability of blood pressure and hemodynamics in African- and European-American youth. Hypertension 2003, 41(6):1196-1201.

6. Levy D, Larson M, Benjamin E, Newton-Cheh C, Wang T, Hwang S-J, Vasan R, Mitchell G: Framingham heart study $100 \mathrm{~K}$ project: genome-wide associations for blood pressure and arterial stiffness. BMC Med Genet 2007, 8(Suppl 1):S3.

7. Tipnis SR, Hooper NM, Hyde R, Karran E, Christie G, Turner AJ: A human homolog of angiotensin-converting enzyme. J Biol Chem 2000, 275(43):33238-33243.

8. Donoghue M, Hsieh F, Baronas E, Godbout K, Gosselin M, Stagliano N, Donovan $M$, Woolf $B$, Robison $K$, Jeyaseelan $R$, et al: A novel angiotensin- 
converting enzyme related carboxypeptidase (ACE2) converts angiotensin I to angiotensin 1-9. Circ Res 2000, 87(5):e1-e9.

9. Crackower MA, Sarao R, Oudit GY, Yagil C, Kozieradzki I, Scanga SE, Oliveirados-Santos AJ, da Costa J, Zhang L, Pei Y, et al: Angiotensin-converting enzyme 2 is an essential regulator of heart function. Nature 2002, 417(6891):822-828.

10. Danilczyk $U$, Penninger JM: Angiotensin-converting enzyme II in the heart and the kidney. Circ Res 2006, 98(4):463-471.

11. Huang W, Yang W, Wang Y, Zhao Q, Gu D, Chen R: Association study of angiotensin-converting enzyme 2 gene (ACE2) polymorphisms and essential hypertension in northern Han Chinese. J Hum Hypertens 2006, 20(12):968-971.

12. Gurley SB, Allred A, Le TH, Griffiths R, Mao L, Philip N, Haystead TA, Donoghue $\mathrm{M}$, Breitbart RE, Acton SL, et al: Altered blood pressure responses and normal cardiac phenotype in ACE2-null mice. $J$ Clin Invest 2006, 116(8):2218-2225.

13. Benjafield AV, Wang WYS, Morris BJ: No association of angiotensinconverting enzyme 2 gene (ACE2) polymorphisms with essential hypertension. Am J Hypertens 2004, 17(7):624-628.

14. Lieb W, Graf J, Götz A, König I, Mayer B, Fischer M, Stritzke J, Hengstenberg C, Holmer S, Döring A, et al: Association of angiotensin-converting enzyme 2 (ACE2) gene polymorphisms with parameters of left ventricular hypertrophy in men. Results of the MONICA Augsburg echocardiographic substudy. J Mol Med (Berl) 2006, 84(1):88-96.

15. Fan $X$, Wang $Y$, Sun $K$, Zhang $W$, Yang $X$, Wang $S$, Zhen $Y$, Wang J, Li W, Han Y, et al: Polymorphisms of ACE2 gene are associated with essential hypertension and antihypertensive effects of Captopril in women. Clin Pharmacol Ther 2007, 82(2):187-196.

16. Yi L, Gu YH, Wang XL, An LZ, Xie XD, Shao W, Ma LY, Fang JR, An YD, Wang $F$, et al: Association of ACE, ACE2 and UTS2 polymorphisms with essential hypertension in Han and Dongxiang populations from Northwestern China. J Int Med Res 2006, 34(3):272-283.

17. O'Loughlin J, Karp I, Koulis T, Paradis G, Difranza J: Determinants of first puff and daily cigarette smoking in adolescents. Am J Epidemiol 2009, 170 (5):585-597.

18. O'Loughlin J, Karp I, Henderson M, Gray-Donald K: Does cigarette use influence adiposity or height in adolescence? Ann Epidemiol 2008, 18(5):395-402.

19. Maximova K, O'Loughlin J, Paradis G, Hanley JA, Lynch J: Declines in physical activity and higher systolic blood pressure in adolescence. Am J Epidemiol 2009, 170(9):1084-1094.

20. Centers for Disease Control and Prevention: A SAS program for the CDC growth charts. Available at http://www.cdc.gov/nccdphp/dnpao/ growthcharts/resources/sas.htm.

21. de Bakker PIW, Yelensky R, Pe'er I, Gabriel SB, Daly MJ, Altshuler D: Efficiency and power in genetic association studies. Nat Genet 2005, 37(11):1217-1223.

22. Barrett JC, Fry B, Maller J, Daly MJ: Haploview: analysis and visualization of LD and haplotype maps. Bioinformatics 2005, 21(2):263-265.

23. Shankar RR, Eckert GJ, Saha C, Tu W, Pratt JH: The change in blood pressure during pubertal growth. J Clin Endocrinol Metab 2005, 90(1):163-167.

24. Maximova K, O'Loughlin J, Paradis G, Hanley JA, Lynch J: Changes in anthropometric characteristics and blood pressure during adolescence. Epidemiology 2010, 21(3):324-331.

25. Fossella J, Sommer T, Fan J, Wu Y, Swanson JM, Pfaff DW, Posner MI: Assessing the molecular genetics of attention networks. BMC Neurosci 2002, 3:14.

26. Sherry ST, Ward MH, Kholodov M, Baker J, Phan L, Smigielski EM, Sirotkin K: dbSNP: the NCBI database of genetic variation. Nucleic Acids Res 2001, 29(1):308-311

27. Cook NR, Ware JH: Design and analysis methods for longitudinal research. Annu Rev Public Health 1983, 4:1-23.

28. Zhao Q, Hixson JE, Rao DC, Gu D, Jaquish CE, Rice T, Shimmin LC, Chen J, Cao J, Kelly TN, et al: Genetic variants in the apelin system and blood pressure responses to dietary sodium interventions: a family-based association study. J Hypertens 2010, 28(4):756-763.

29. Zhu M, Zhao S: Candidate gene identification approach: progress and challenges. Int J Biol Sci 2007, 3(7):420-427.
30. Jorgensen TJ, Ruczinski I, Kessing B, Smith MW, Shugart YY, Alberg AJ: Hypothesis-driven candidate gene association studies: practical design and analytical considerations. Am J Epidemio/ 2009, 170(8):986-993.

31. Clayton D: Testing for association on the $\mathrm{X}$ chromosome. Biostatistics (Oxford, England) 2008, 9(4):593-600.

32. Clayton DG: Sex chromosomes and genetic association studies. Gen Med 2009, 1(11):110.

33. Hickey PF, Bahlo M: $\mathrm{X}$ chromosome association testing in genome wide association studies. Gen Epidemiol 2011, 35(7):664-670.

34. Brown MJ: Hypertension and ethnic group. BMJ 2006, 332(7545):833-836.

doi:10.1186/1471-2350-14-117

Cite this article as: Malard et al:: The association between the angiotensin-converting enzyme-2 gene and blood pressure in a cohort study of adolescents. BMC Medical Genetics 2013 14:117.

\section{Submit your next manuscript to BioMed Central and take full advantage of:}

- Convenient online submission

- Thorough peer review

- No space constraints or color figure charges

- Immediate publication on acceptance

- Inclusion in PubMed, CAS, Scopus and Google Scholar

- Research which is freely available for redistribution
C Biomed Central 